\title{
Clinical evaluation of a matrix metalloproteinase-12 cleaved fragment of titin as a cardiovascular serological biomarker
}

\author{
Efstathios Vassiliadis ${ }^{1,2^{*}}$, Lars M Rasmussen ${ }^{3}$, Inger Byrjalsen ${ }^{1}$, Dorthe Vang Larsen ${ }^{1}$, Rajiv Chaturvedi ${ }^{4}$, \\ Susanne Hosbond ${ }^{5}$, Lotte Saabye ${ }^{5}$, Axel CP Diederichsen ${ }^{5}$, Federica Genovese ${ }^{1}$, Kevin L Duffin ${ }^{6}$, Qinlong Zheng ${ }^{7}$, \\ Xiaoliang Chen ${ }^{7}$, Diana J Leeming ${ }^{1}$, Claus Christiansen ${ }^{1}$ and Morten A Karsdal ${ }^{1,3}$
}

\begin{abstract}
Background: Titin is a muscle-specific protein found in cardiac and skeletal muscles which is responsible for restoring passive tension. Levels and functioning of titin have been shown to be affected by cardiac damage. Due to the inherent difficulty of measuring titin levels in vivo in a clinical setting, we aimed to develop an assay that could reliably measure fragments of degraded titin in serum and potentially be used in the assessment of cardiac muscle damage.

Methods: A competitive ELISA was developed to specifically measure levels of the titin sequence 12670' NVTVEARLIK 12679', derived by the degradation of titin by matrix metalloproteinase (MMP)-12. Serum samples from 90 individuals were divided into 3 equally sized groups. One group had been diagnosed with acute myocardial infarction (AMI) while the remaining two were asymptomatic individuals either with CT-scan signs of coronary calcium (CT-plusCa) or without coronary calcium (CT-noCa).

Results: Mean geometric levels of the titin fragment in the CT-noCa group were $506.5 \mathrm{ng} / \mathrm{ml}( \pm 43.88)$. The CT-plusCa group showed $50.6 \%$ higher levels of the marker $[763 \mathrm{ng} / \mathrm{ml}( \pm 90.14)](P<0.05)$. AMl patients showed $56.3 \%$ higher levels [792 ng/ml $( \pm 149)](P<0.05)$.
\end{abstract}

Conclusions: The titin-12670 fragment is present in both individuals with undiagnosed and diagnosed CVD. The statistically significant increase in level of the marker in the AMl group is indicative that this neoepitope biomarker may be a useful serological marker in AMI.

Keywords: Titin, CVD, MMP-12, Cardiovascular, Acute myocardial infarction, Biomarker, Neoepitope

\section{Background}

Titin, also known as connectin, is a sarcomeric protein expressed in cardiac and skeletal muscle. It is the largest known protein in nature, with a molecular weight of up to $3700 \mathrm{kDa}$ [1]. Its main function in the heart is to act as a long molecular spring by restoring passive tension during myocardial stretch and enhancing or terminating active force thus regulating the Frank-Starling mechanism of the heart [2-5]. Distinct passive stress differences

\footnotetext{
* Correspondence: eva@nordicbioscience.com

${ }^{1}$ Nordic Bioscience A/S, Herlev Hovedgade 207, DK-2730 Herlev, Denmark

${ }^{2}$ School of Endocrinology, University of Southern Denmark, Odense, Denmark

Full list of author information is available at the end of the article
}

have been recorded between cardiac and skeletal muscles [6]. Titin has two isoforms that are co-expressed in the sarcomere, the N2A which is the larger of the two and is found in both skeletal and myocardial muscle, and the $\mathrm{N} 2 \mathrm{~B}$ isoform which is smaller, stiffer and is found in cardiac muscle [1,7-12]. Due to the different stiffness in titin's isoforms, it has been proposed that the adaptive or maladaptive ratio alteration between the two isoforms could affect its myocardial contractile properties [4,7,13-17]. Isoform modifications and ratio alterations were first described in animal models while clinical studies have also reported isoform changes during dilated cardiomyopathy (DCM), aortic stenosis (AS), diastolic heart failure (DHF) and ischemic heart disease (IHD)

\section{Ciomed Central}


[16,18-21]. The main limitation of studies of titin lies in the methods for detecting and quantifying titin levels. The studies rely on invasive tissue extractions which are then analysed by methods such as immunoblotting and gel electrophoresis.

Extracellular matrix (ECM) components are degraded by a number of different proteases including matrix metalloproteinases (MMPs). MMP-degradation of proteins generates specific cleavage sites on fragments which in turn enable the development of new epitopes. Our group previously discussed neoepitopes that may have potential utility as biomarkers of unbalanced ECM remodeling in a number of different pathologies and can be measured in biological fluids such as serum, plasma and urine [22-27]. Key benefits of measuring biomarkers in body fluids are that this process is non-invasive and, because the specific neoepitopes represent a unique 'fingerprint' of the proteolytic cleavage of the protein, they identify the specific tissue being turned over and also detect whether the tissue is diseased or healthy. Since pathology-related cardiac remodeling is initiated before clinical onset and appearance of any symptoms [28], biomarkers that indicate abnormal remodeling could be utilised for early diagnosis of cardiovascular disease (CVD). The cardiac-specific markers troponin I and $\mathrm{T}$ (cTnI \& cTnT) are already used to closely monitor myocardial damage and related pathological events [29-33]. Titin has been previously shown to be degraded by MMP-2 localised in the Z-disk of the cardiac sarcomere $[34,35]$. However to our knowledge no biomarkers based on titin-relevant neoepitopes resulting from the degradation activity of either MMP-2 or other metalloproteinases have been described.

During digestion of human tissue by an array of exogenous metalloproteinases, a large number of proteolyzed peptides have been identified using mass spectrometry [36]. Among these, a titin specific fragment 12670' NVTVEARLIK 12679' was identified to have been cleaved specifically by MMP-12. Proteomic analysis revealed that the sequence is located in the IG domain within the distal tandem IG segment and is homologous only in humans and mice. Even though at least in murine models MMPs and MMP-12 in particular have been implicated in cardiovascular events such as atherogenenesis [37], the only MMP previously described to have a direct effect on titin degradation is MMP-2, which contributes to titin degradation in ischemic and reperfusionrelated events [34,37].

We hypothesized that the MMP-12 specific fragment of titin could be potentially useful for monitoring pathologic cardiovascular events and thus as a biomarker. Our hypothesis was that titin degradation fragments may be released and found in the circulation, in a similar way as adjacent proteins in the myocyte, such as troponin, are released and measured, and that the level of titin fragments may be associated to the degree of myocyte damage. Examples of cardiac markers based on troponin, a protein which is in close proximity to and interacts with titin, indicate that release of myocyterelated protein remodeling fragments into the circulation can provide accurate markers of pathology-related remodeling. We investigated this hypothesis by developing a monoclonal antibody and a serum-based assay for the identification of the titin fragment degenerated specifically by MMP-12. We used the assay in three well- characterised populations with different degrees of heart disease, but with comparable gender- and age-composition. The samples were collected by the same hospital staff using standard procedures, hence adding low biological variance and increased clinical significance.

\section{Methods}

\section{Reagents}

All reagents used for experiments were standard highquality chemicals from Merck (Whitehouse Station, NJ, USA) and Sigma Aldrich (St. Louis, MO, USA). The synthetic peptides used for monoclonal antibody production were purchased from the Chinese Peptide Company, Beijing, China.

\section{Selection of the peptide for immunization}

The amino acid sequence selected for the assay was chosen from mass spectrometry performed on human tissue [36]. Peptide fragments were identified using the Uniprot database, with the accession number COJYZ2. The sequence NVTVEARLIK located between amino acid position 12670' and 12679' (titin) was selected as the immunogen. The first 10 amino acids of each free end of the sequences identified were regarded as a target sequence. All relevant sequences were analyzed for homology and then blasted for homology using the NPS@: network protein sequence analysis [38]. The sequence was identified by Uniprot and PBIL (Pole Bio Informatique Lyonnais) network protein sequence analysis in UNIPROT-SWISSPROT databases and was found to be unique to human and mouse titin. The full 10- amino acid sequence was also blasted with 1 and 2 mismatched amino acids as well as with 95\%, 90\%, 85\%, 80\%, 75\%, $70 \%$ similarity levels. In all cases the 10 amino acid sequence was found to be unique for human and mouse titin. The selected sequence was also found to be present in 6 out of 8 titin isoforms produced by alternative splicing. These were isoforms 3 (small cardiac N2B), 7 (cardiac novex-2) and 8 (cardiac novex-1) that are known to be present in cardiac muscle (Uniprot accession numbers Q8WZ42-3, Q8WZ42-7 and Q8WZ42-8 respectively) and isoforms 2, 4 and 5 (Uniprot accession numbers Q8WZ42-2, Q8WZ42-4 and Q8WZ42-5 respectively) [39]. 
The 6 amino acids from the $C$ terminal end of the selected peptide (12675' EARLIK 12679') were also blasted using NPS to assess and identify the sequence similarity with potential cross-reactive sequences found in the circulation. This only revealed a similarity with a sequence found in sterile, alpha motif domaincontaining protein, while the remaining hits were found to be titin-specific.

\section{Immunization procedure}

Six 4-6 week old Balb/C mice were immunized subcutaneously in the abdomen with $200 \mu \mathrm{L}$ emulsified antigen (50 $\mu \mathrm{g}$ per immunization), using Freund's incomplete adjuvant (KLH-NVTVEARLIK). Immunizations were performed at two-week intervals until stable titre levels were obtained. At each bleeding, the serum antibody titre was measured and the mice with the highest antibody titre and best reactivity towards serum and urine were selected for fusion. The selected mice were boosted intravenously with $50 \mu \mathrm{g}$ immunogen in $100 \mu \mathrm{L} 0.9 \%$ sodium chloride solution three days before surgical removal of the spleen for cell fusion. The study was approved by the Beijing laboratory animal administration office under approval number 200911250.

\section{Fusion and antibody screening}

The fusion procedure has been described elsewhere [40]. Briefly, mouse spleen cells were fused with SP2/0 myeloma fusion partner cells. The hybridoma cells were cloned using a limiting dilution method and transferred into 96-well microtiter plates for further growth. Standard limiting dilution was used to promote monoclonal growth. Supernatants were screened using an indirect ELISA, while the biotinylated peptide Biotin-NVTVEARLIK was used as a catcher peptide on streptavidin-coated microtitre plates.

\section{Characterization of clones}

Native reactivity and peptide-binding of the monoclonal antibodies in human serum was evaluated using a preliminary ELISA with a $10 \mathrm{ng} / \mathrm{mL}$ biotinylated peptide coater on a streptavidin-coated microtitre plate and the supernatant from the growing monoclonal hybridoma. Clone specificity was tested against a free synthetic peptide (NVTVEARLIK) and a non-sense synthetic peptide sequence. Isotyping of the monoclonal antibodies was performed using the Clonotyping System-HRP kit, cat.5300-05 (Southern Biotech, Birmingham, AL, USA). The selected clones were purified using Protein G columns according to the manufacturer's instructions and dialysed (GE Healthcare Life Science, Little Chalfont, Buckinghamshire, UK).

\section{MMP-12 titin assay protocol}

The following competitive ELISA protocol was optimised for use with the MMP-12 titin monoclonal antibody. The selected monoclonal antibodies were labelled with horseradish peroxidase (HRP) using the LightningLink Horseradish Peroxidase (HRP) antibody labelling kit according to the manufacturer's instructions (Innovabioscience, Babraham, Cambridge, UK). A 96-well streptavidin plate (Roche Diagnostics, Basel, Switzerland) was coated with $1.3 \mathrm{ng}$ of the biotinylated synthetic peptide, Biotin-CGG-NVTVEARLIK, dissolved in assay buffer $50 \mathrm{mM}$ Tris BTB (p.H 8 at $20^{\circ} \mathrm{C}$ ) and incubated for 30 minutes at $20^{\circ} \mathrm{C}$. $20 \mu \mathrm{L}$ of the peptide calibrator or sample were added to appropriate wells, followed by $100 \mu \mathrm{L}$ of $40 \mathrm{ng} / \mathrm{ml}$ conjugated monoclonal antibody, and incubated for 1 hour at $4^{\circ} \mathrm{C}$. Finally, $100 \mu \mathrm{L}$ tetramethyl benzinidine (TMB) (Kem-En-Tec cat.438OH, Taastrup, Denmark) was added, and the plate was incubated for 15 minutes at $20^{\circ} \mathrm{C}$ in the dark. All of the above incubation steps included shaking at $300 \mathrm{rpm}$. After each incubation step the plate was washed five times in washing buffer $(20 \mathrm{mM}$ Tris, $50 \mathrm{mM} \mathrm{NaCl}, \mathrm{pH}$ 7.2). The TMB reaction was stopped by adding $100-\mu \mathrm{L}$ of stopping solution $(1 \% \mathrm{HCl})$ and measured at $450 \mathrm{~nm}$ with $650 \mathrm{~nm}$ as the reference. A calibration curve was plotted using a 4-parametric mathematical fit model with a starting concentration of $1000 \mathrm{ng}$ for the standard peptide following a 2-fold dilution.

\section{Technical evaluation}

From 2-fold dilutions of human serum, linearity was calculated as a percentage of recovery of the $100 \%$ sample. The lower limit of detection (LLD) was determined from 21 zero samples (i.e. buffer) and calculated as the mean $+3 x$ standard deviations. The inter- and intraassay variation was determined by 10 independent runs of six quality control (QC) human serum and mouse heparin plasma samples run in duplicate.

\section{Tissue specificity}

Cardiac and skeletal muscle where collected from an adult mouse and frozen in liquid nitrogen. Mouse tissue was selected for the specificity test because of the $100 \%$ homology of the selected antibody sequence between human and mouse, and because of the lack of available human tissue samples for both cardiac and corresponding skeletal tissue. Titin was extracted according to the method described by Granzier and Irving [6]. Briefly, the tissues were pulverized to a fine powder using a mortar and a pestle bathed in liquid nitrogen, and then rapidly added to 9 volumes of hot solubilization buffer $(50 \mathrm{mM}$ Tris- $\mathrm{HCl}, 2 \%$ SDS, 10\% glycerol, $80 \mathrm{mM} \mathrm{DTT)}$ and homogenized for 90 seconds in a $90-95^{\circ} \mathrm{C}$ water bath. 
The extracted samples were then immediately cooled down and stored at $-20^{\circ} \mathrm{C}$.

A buffer exchange was performed using Vivaspin-2 3000 MWCO PES columns (Sartorius Stedim Biotech, Goettingen, Germany) against PBS $1 \mathrm{X}$ and the protein concentration was measured using the NanoDrop ND1000 spectrophotometer (Thermo Scientific, Waltham, Massachusetts, USA). MMP-12 derived titin fragments were measured in the extracted samples using the ELISA assay described above.

\section{Patients and sample collection \\ Patients}

As described in detail below, our study is a case-control study, in which we selected and grouped individuals on the basis of prior knowledge about the presence or absence of well-defined manifestations of ischemic heart disease. The groups were chosen for the sole purpose of investigating connections between biomarkers and differences in the degree or type of ischemic heart disease. Individuals from larger ongoing studies were selected to ensure this analysis compared populations with a similar gender- and age- distribution. Importantly, pre-analytical conditions were similar in all groups.

Ninety individuals with different degrees of atherosclerotic heart disease, were selected from larger studies (DANRISK and DEFAMI), which were actively enrolling patients at Odense University Hospital in Denmark during the same time period. The individuals were divided into three groups of 30 . One group consisted of subjects without previous CVD and no coronary calcium detectable on a CT scan (CT-noCa); another 30 asymptomatic subjects from the DANRISK study had no previous CVD but were diagnosed with subclinical CVD due to their severe subclinical coronary calcium shown on CT scans (CT-plusCa); and the remaining 30 patients had acute AMI, and were from the DEFAMI study (Table 1). All samples from these individuals were pre-analytically handled and stored by the analytical unit at Odense University Hospital.

In the DANRISK-study [41], a random sample of 1825 middle-aged men and women was in 2009 invited for screening for coronary disease. Screening included a cardiac CT scan, followed by an estimation of the amount of coronary calcium using the Agatston score. Of the invited subjects, 1257 were considered for inclusion in the study. From this group were excluded those with prior ischemic heart disease or diabetes mellitus $(n=100)$, leaving a total of 1157 patients for analysis. Among the individuals aged 60 years $(n=647)$, we selected 30 patients without coronary calcium (CT-noCa) and another 30 with an Agatston score $\geq 400$ (CT-plusCa).

In the DEFAMI-study, all patients admitted between October 12009 and April 302010 at any clinical department at Odense University Hospital and having troponin
Table 1 Demographics of each group in the study, mean values and standard deviation

\begin{tabular}{|c|c|c|c|c|}
\hline & $\begin{array}{l}\text { CT-noCa } \\
\mathrm{n}=30\end{array}$ & $\begin{array}{l}\text { CT-plusCa } \\
\mathrm{n}=30\end{array}$ & $\begin{array}{l}\text { AMI } \\
\mathrm{n}=30\end{array}$ & $\begin{array}{l}\text { ANOVA } \\
\text { p-value }\end{array}$ \\
\hline \multirow[t]{2}{*}{ Age (yrs.) } & 60.2 & 60.3 & $64.5^{* *}$ & 0.001 \\
\hline & {$[59.8 ; 60.7]$} & {$[60.0 ; 60.6]$} & {$[56.0 ; 73.0]$} & \\
\hline \multirow{2}{*}{$\begin{array}{l}\text { Triglycerides }{ }^{A} \\
(\mathrm{mmol} / \mathrm{L})\end{array}$} & 1.57 & 1.49 & 1.30 & 0.52 \\
\hline & {$[0.85 ; 2.93]$} & {$[1.00 ; 2.21]$} & {$[0.78 ; 2.19]$} & \\
\hline \multirow{2}{*}{$\begin{array}{l}\text { HDL Cholesterol }{ }^{\mathrm{A}} \\
(\mathrm{mmol} / \mathrm{L})\end{array}$} & 1.28 & 1.36 & 1.13 & 0.07 \\
\hline & {$[0.93 ; 1.75]$} & {$[0.98 ; 1.89]$} & {$[0.83 ; 1.52]$} & \\
\hline \multirow{2}{*}{$\begin{array}{l}\text { LDL Cholesterol } \\
(\mathrm{mmol} / \mathrm{L})\end{array}$} & 3.14 & 3.26 & 2.93 & 0.47 \\
\hline & {$[2.13 ; 4.16]$} & [2.29; 4.22] & {$[1.74 ; 4.11]$} & \\
\hline \multirow{2}{*}{$\begin{array}{l}\text { Total Cholesterol } \\
\text { (mmol/L) }\end{array}$} & 5.25 & 5.47 & 4.74 & 0.07 \\
\hline & {$[4.06 ; 6.43]$} & {$[4.29 ; 6.64]$} & {$[3.44 ; 6.04]$} & \\
\hline \multirow{2}{*}{$\begin{array}{l}\text { Systolic Blood } \\
\text { Pressure }(\mathrm{mm} \mathrm{Hg})\end{array}$} & 145 & 147 & $159^{*}$ & 0.03 \\
\hline & {$[131 ; 159]$} & {$[128 ; 167]$} & {$[132 ; 187]$} & \\
\hline \multirow{2}{*}{$\begin{array}{l}\text { Diastolic Blood } \\
\text { Pressure }(\mathrm{mm} \mathrm{Hg})\end{array}$} & 86 & 85 & 88 & 0.80 \\
\hline & {$[76 ; 95]$} & {$[75 ; 96]$} & {$[72 ; 103]$} & \\
\hline \multirow[t]{2}{*}{ Agatston score } & - & 1299 & - & - \\
\hline & & {$[717 ; 2352]$} & & \\
\hline \multirow[t]{2}{*}{ Heartscore $^{A}$} & 5.29 & 6.38 & 8.00 & 0.07 \\
\hline & {$[2.89 ; 9.68]$} & {$[2.96 ; 13.7]$} & {$[4.01 ; 16.0]$} & \\
\hline \multirow[t]{2}{*}{ Osteopontin ${ }^{A}$} & 83 & 99 & 111 & 0.08 \\
\hline & {$[52 ; 132]$} & {$[67 ; 145]$} & {$[61 ; 201]$} & \\
\hline \multirow[t]{2}{*}{ Osteoprotegerin ${ }^{A}$} & 1583 & 1704 & $2170^{* *}$ & 0.0007 \\
\hline & {$[1144 ; 2189]$} & {$[1335 ; 2175]$} & {$[1487 ; 3168]$} & \\
\hline \multirow[t]{2}{*}{ Fibulin $^{\mathrm{A}}$} & 63 & 63 & $78^{* *}$ & 0.0006 \\
\hline & {$[49 ; 80]$} & {$[50 ; 80]$} & {$[62 ; 99]$} & \\
\hline
\end{tabular}

CT-noCa: Asymptomatic individuals without detectable coronary calcium; CT-plusCa: Asymptomatic CVD with high coronary calcium; AMI (Acute Myocardial Infarction): patients with NSTEMI-ACS. Mean values [+/- STD)], ${ }^{A}$ Geometric mean values [+/- STD)], and p-value from one-way of analysis of variance (ANOVA). The level of significance of $p$-values from comparison of each group against the control group ' $\mathrm{CT}$-noCa'was adjusted for multiple comparisons by the method of Dunnet $\left({ }^{*}: p<0.05 ;{ }^{* *}: p<0.01 ;{ }^{* * *}: p<0.001\right)$.

analysis performed because of suspected acute coronary syndrome were enrolled. Blood sampling was done as part of a large Biobank within the first 24 hours of symptom onset. Of the 822 patients in the DEFAMI study, 30 individuals with non-ST elevation myocardial infarction (NSTEMI, $\mathrm{n}=24$ ) or unstable angina $(\mathrm{n}=6)$ were selected to participate in the current study. Their age was approximately 60 years and their gender distribution matched the two above-mentioned DANRISK subgroups. NSTEMI was defined as increased TnI levels above $0.03 \mu \mathrm{g} / \mathrm{l}$, but ECG without ST-elevations. Unstable angina was defined as chest pain occurring during rest or minimal physical exertion. The mean TnI level in the NSTEMI-ACS group was $0.62 \mu \mathrm{g} / \mathrm{l}$ (range 0.01-5.23 $\mu \mathrm{g} / \mathrm{l})$. The NSTEMI-ACS patient group was chosen because in these patients it was possible to 
obtain blood samples before medication with heparin or other interventions for acute myocardial infarction, which could interfere with biochemical assays.

In these three patient groups, hypertension was defined as the use of antihypertensive medical treatment and diabetes as the use of anti-diabetic medication. The patients were considered to have hypercholesterolemia if the diagnosis was stated in the patient file or if the patient was taking cholesterol-lowering treatment.

Systolic and diastolic blood pressure was measured on the same day as blood sampling. Agatston score was calculated in the two groups of patients undergoing cardiac CT. Troponin in the NSTEMI-ACS group was measured prior to the blood sampling. Total cholesterol, LDL, HDL and triglycerides were measured prior to the blood sampling. Blood samples were drawn in tubes with EDTA and centrifuged at $200 \mathrm{~g}$ for $10 \mathrm{~min}$. Plasma was stored at $-80^{\circ} \mathrm{C}$ until biochemical analysis.

Protocols for all three studies at Odense University Hospital were approved by the Regional Ethics Committee before the initiation of the trials. Informed consent was obtained from all participating patients.

\section{Statistical analysis}

The data of HDL cholesterol, heartscore, the ratio between LDL and HDL cholesterol, and levels of HDL triglycerides, osteoprotegrin (OPG), fibulin, osteopontin (OPN) and titin were logarithmically transformed to obtain symmetry of variance. One-way analysis of variance (ANOVA) was used for comparison among groups. If ANOVA revealed a statistically significant difference between groups, each of the groups (CT-plusCa and AMI) was compared with the control group (CT-noCa) with the level of significance adjusted for multiple comparisons by the method of Dunnet. For each group of CT-plusCa and AMI, the markers showing a statistically significant difference between groups in the ANOVA were further investigated by ROC curve against the CT-noCa group.

The relationship between titin and other factors (triglycerides, LDL-cholesterol, HDL-cholesterol, total cholesterol, systolic blood pressure, diastolic blood pressure, and age) was investigated by univariate linear regression analysis and multivariate analysis of variance using the general linear model procedure. The diagnostic value of the assay was calculated by receiver-operating characteristic (ROC) curve plots.

The Statistical Analysis System (SAS Institute, Cary, NC, USA) was used for the analyses. The level chosen to indicate statistical significance was $5 \%$.

\section{Results}

\section{Demographics}

Key demographic information and biochemical measurements are presented in Table 1.

\section{Clone characterization \& tissue specificity}

The clone selected for ELISA development was determined to be the IgG1 subtype. The native reaction of this clone was high against human serum. The clone was found to react specifically to the target sequence and not the elongated peptide (Figure 1). The clone showed higher signal inhibition towards mouse cardiac muscle (51\% inhibition) than to skeletal mouse extracts (21\% inhibition).

\section{Technical evaluation}

The typical standard curve is presented in Figure 1, showing a 4-parametric fit for the assay. The lower limit of detection for the assay was $9.78 \mathrm{ng} / \mathrm{mL}$. Dilution recovery was found to be within $100 \pm 15 \%$. The interand intra-assay variation was found to be 4.33 and $5.49 \%$ respectively.

\section{Clinical cohort}

A statistically significant increase in the marker was measured in CT-plusCa and AMI patient groups (Table 2). The geometric mean level of the MMP-12 degraded titin fragment in the $\mathrm{CT}$-noCa group was $506.5 \mathrm{ng} / \mathrm{ml}$. In the AMI patient group, the mean value was $792 \mathrm{ng} / \mathrm{ml}(56.3 \%$ increase, $\mathrm{P}<0.05)$, and in the CT-plusCa group $763 \mathrm{ng} / \mathrm{ml}( \pm 90.14)$ (43\% increase,

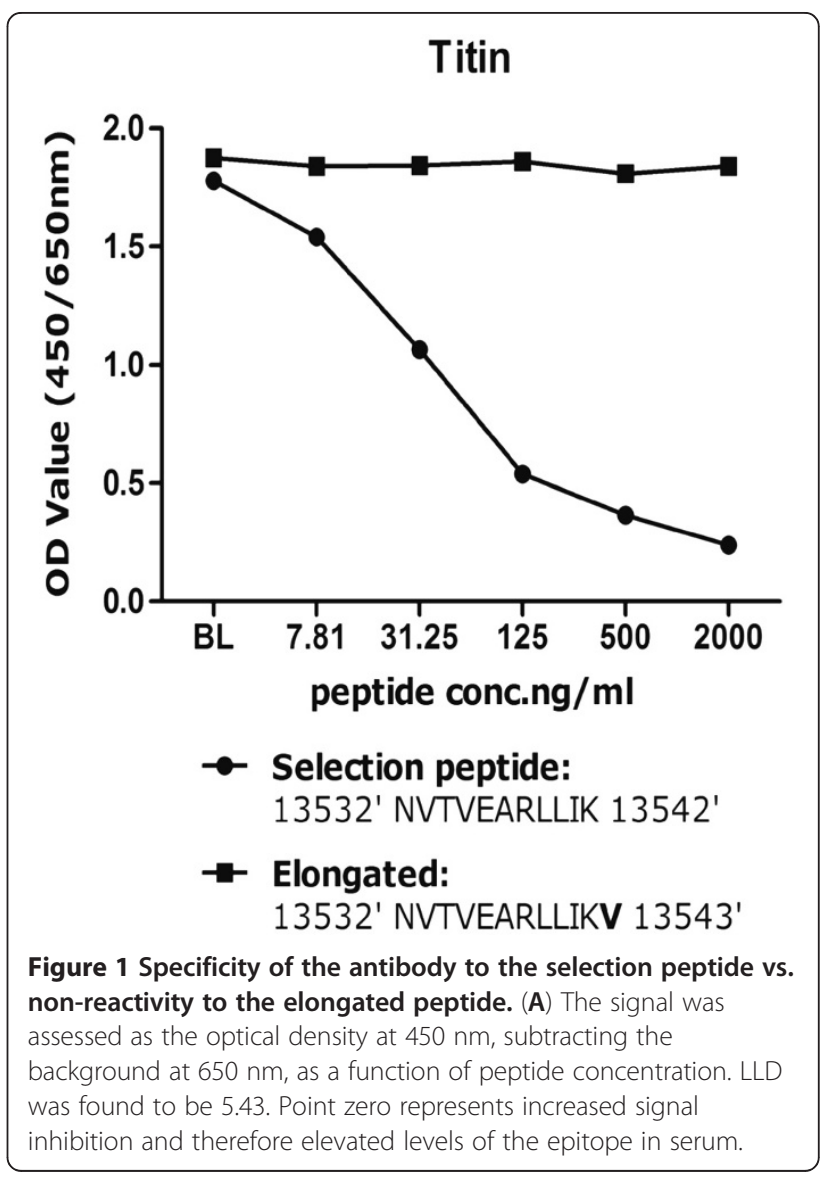


Table 2 Comparison of levels of MMP-12-generated fragments of titin in the clinical cohort

\begin{tabular}{llll}
\hline & $\begin{array}{l}\text { CT-noCa } \\
\mathbf{n = 3 0}\end{array}$ & $\begin{array}{l}\text { CT-plusCa } \\
\mathbf{n = 3 0}\end{array}$ & $\begin{array}{l}\text { AMI } \\
\mathbf{n = 3 0}\end{array}$ \\
\hline Geometric mean value $(\mathrm{ng} / \mathrm{ml})$ & 506.5 & 759.7 & 792.5 \\
\hline SEM & 43.88 & 90.14 & 149.5 \\
\hline \% Increase & Control & $43 \%$ & $56.3 \%$ \\
\hline P Value & Control & 0.03 & 0.02 \\
\hline
\end{tabular}

$\mathrm{P}<0.05$ ) (Figure 2). Although Levene's test for homogeneity of variance was non-significant $(p=0.07)$ there was a tendency to higher variance in the CT-plusCa group (standard deviation 74\%); AMI (standard deviation $97 \%$ ) as compared with the CT-noCa group (standard deviation 54\%).

The highest sensitivity and specificity of the assay was found at the cut-off value of $545 \mathrm{ng} / \mathrm{ml}$. At this value the sensitivity and specificity were $73.3 \%$ and $63.3 \%$ respectively for the CT-plusCa group, while the positive predictive value (PPV) and negative predictive value (NPV) were $64.7 \%$ and $68 \%$ respectively. In the AMI group, sensitivity and specificity was $67.7 \%$ and $63.3 \%$ respectively, while the PPV and NPV were $65.6 \%$ and $65 \%$.

\section{ROC values of the assay}

The assay was found to have a highly significant ROC value for all patient groups (Table 3).

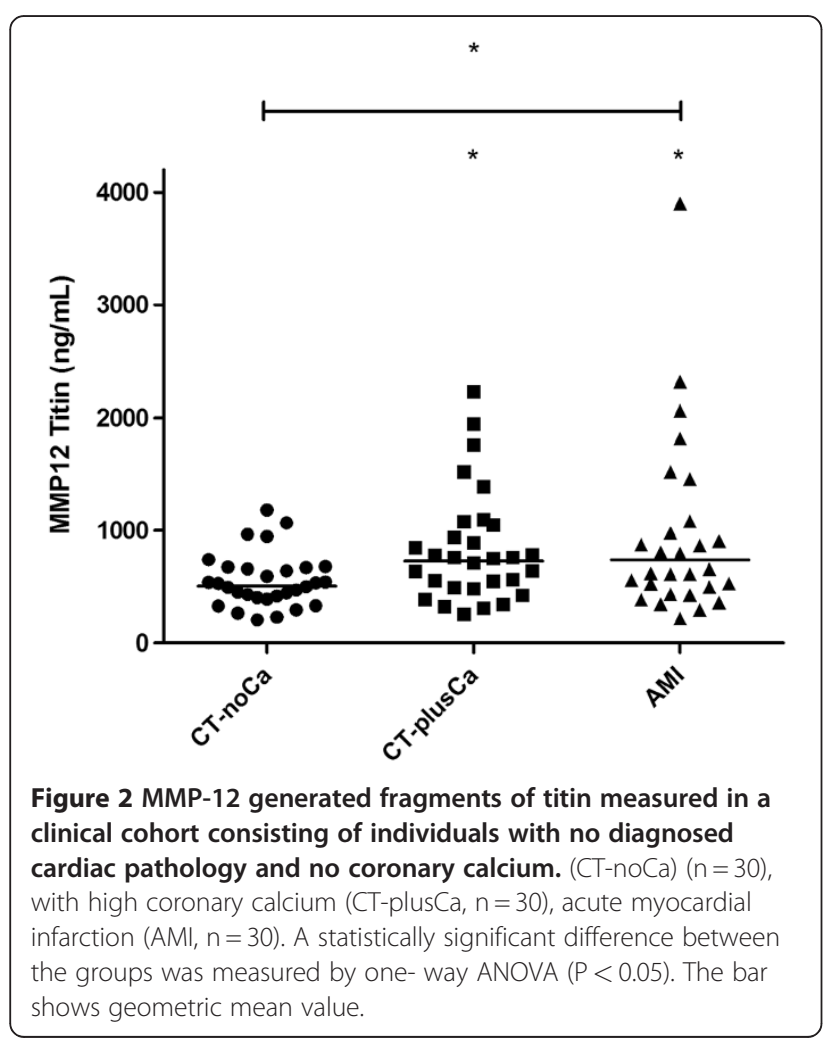

Table 3 ROC values of the assay measuring MMP-12 generated titin fragments, vs. other known cardiac markers

\begin{tabular}{lll}
\hline CT-noCa & Vs. CT-plusCa & Vs. AMI \\
\hline Systolic Blood Pressure & 0.54 & $0.70^{* *}$ \\
\hline Total Cholesterol & 0.55 & 0.61 \\
\hline LDL & 0.53 & 0.55 \\
\hline Heart score & 0.57 & $0.70^{* *}$ \\
\hline Osteoprotegerin & 0.58 & $0.73^{* * *}$ \\
\hline Fibulin-1 & 0.49 & $0.74^{* * *}$ \\
\hline Osteopontin & $0.64^{*}$ & $0.64^{*}$ \\
\hline MMP12-Titin & $\mathbf{0 . 6 9}^{* *}$ & $\mathbf{0 . 6 5}$ \\
\hline
\end{tabular}

\section{Relationship between titin and other factors}

The marker showed no significant correlation with any other physiological measurements. The relationship between titin and the physiological characteristics of triglycerides, LDL-cholesterol, HDL-cholesterol, total cholesterol, systolic blood pressure, diastolic blood pressure, and age was statistically non-significant both in the separate univariate linear regression analysis and in multivariate analysis of variance (Wilks Lambda criteria: CT-noCa: $\mathrm{p}=0.79$; CT-plusCa: $\mathrm{p}=0.39$; AMI: $\mathrm{p}=0.74$ ). The level of titin in the AMI patients also showed no significant correlation with the troponin I levels.

\section{Discussion}

The study relied on a well-characterised cohort in which all samples were collected under the same standard operating procedures. The study presents for the first time an assay for in vivo detection of titin degradation fragments that have been associated with abnormal remodeling and cardiac damage. The assay had a good native reactivity in human serum and was technically robust with acceptable inter- and intra-assay variation, dilution recovery and a low limit of detection. The assay detected statistically significant increased levels of the biomarker in all the pathology-related clinical cohorts when compared with non-diseased individuals. The assay has shown increased and statistically significant ROC values for all cohorts. Unlike all but one of the other known cardiac markers measured in the cohorts, the titin degradation marker showed a statistically significant elevation with high ROC values in the CTplusCa and AMI groups (Table 3). With the exception of osteopontin, no other marker showed a high ROC and significant $\mathrm{p}$-value in the group of CVD asymptomatic individuals with increased coronary calcium levels (CTplusCa). We believe that the raised titin levels may be a tissue-specific indication of the underlying remodeling processes occurring in the myocyte prior to injury and during pathology development which could be of additional value for early diagnosis of CVD asymptomatic 
patients. However, a longitudinal study and measurement of the MMP-12 titin marker at a later time point in the development of CVD could be of great value to determine the validity of this hypothesis.

The statistically significant increase in the marker levels in the AMI group may be related to the increased macrophage levels found following AMI at the injury site. Macrophages are implicated in all components of AMI response, which include inflammation, scar formation and remodeling [42]. As will be discussed later, macrophages are the main source of MMP-12 in adult tissue which participates in inflammatory processes and ECMR. Hence, increased levels of MMP-12 or other macrophage-derived proteases acting on the infarcted myocardium may be responsible for protease-driven myocardial remodeling. The raised MMP-12 levels are thought to increase degradation of titin at the site of injury, with the titin fragments indicating myocardial injury.

The marker was found not to be correlated with age, LDL, HDL, total cholesterol, triglycerides, BT systolic or diastolic pressure levels which implies that the titin fragment levels are independent of these markers. The lack of correlation may be also attributed to the fact that this assay measures an MMP-degraded fragment of titin and not total protein, as do tests for the above-mentioned biochemical measurements. We believe that the fact that the marker does not directly correlate with the above measurements is not alarming, because the specific titin marker does not measure total protein levels as the above measurements but rather the increased titin degradation levels which results from chronic abnormal remodeling. Further validation of this finding in additional, larger clinical cohorts, could potentially validate this titin marker's utilisation in clinical prediction models as an additional independent risk factor to diagnose asymptomatic patients. The marker should be further evaluated for all criteria set for novel cardiovascular risk factors which include its reliability, sensitivity, availability/practicality, clinical significance and value of independent information on prognosis $[43,44]$.

To date there is no adequate relevant literature regarding the specific activity of MMP-12 - a human macrophage elastase - and its effect on cardiac remodeling during either physiology or pathology. Human and animal studies have established the presence and activity of MMPs such as $-2,-9$ and -7 on cardiac-related remodeling [37,45-52]. Whether the acknowledged MMP-driven activity is dependent on tissue inhibitors of metalloproteinases (TIMPs) or not remains unclear [53]. Even though there is evidence of MMP-12 activity in cardiovascular pathologies such as aortic aneurysms and atherosclerosis [54-58], to our knowledge there is no pre-existing evidence directly implicating MMP-12 activity in cardiac remodeling. The main MMP-12 source in adult tissue is macrophages which have been reported to be an active participant in inflammatory processes and vascular extracellular matrix remodeling [59]. The role of MMP-12 in cardiac pathology is not well understood. Benoit et al. showed that MMP-12 expression was increased in cardiac valves taken from patients suffering from infective endocarditis [60].

Biochemical markers consisting of protein fragments from matrix remodeling degradation may be informative of disease pathology and progression, which in turn may be useful for diagnostic and prognostic purposes. These markers could potentially detect changes resulting from intervention strategies and serve as surrogate markers of drug efficacy [61]. The continuous search and utilisation of cardiac specific markers such as cTnI \& cTnT, that can provide additional information, could be of real clinical value for improved patient care by aiding an early diagnosis, prognosis risk stratification, therapy selection and close monitoring of patients [29]. The described fragment of titin should be further evaluated in additional clinical cohorts in a longitudinal study in which other cardiac markers such as cTnI \& cTnT will also be measured in order to facilitate its categorisation according to the BIPED criteria [22].

The main limitations of this study include the absence of direct quantitative measurements of MMP-12 and other relevant protease levels in the tissue from the clinical cohorts and thus a correlation between the levels of the titin fragment and MMP-12 activity. It should also be noted that we selected and grouped individuals on the basis of knowledge about the presence or absence of well-defined manifestations of ischemic heart disease and to match the groups for age- and gender distributions. However, it is important to note that each group does not necessary reflect all patients with this disease, for example the AMI group, which only included nonSTEMI patients. Additionally, the study lacked a detailed assessment of the troponin $\mathrm{C}$ and I levels in all patients. Due to their position in the myocyte and their vicinity to the titin location, troponin $\mathrm{C}$ and I could have been tested for their correlation to titin. A further limitation is that even though the described fragment is derived from MMP-12 activity in vitro, other proteases or a combination of proteases could potentially generate this fragment in vivo. The fragment's specificity for cardiac tissue was evaluated in mouse tissue, due to the sequence homology between human and mouse, but in future an assessment in human tissue could provide more clinically reliable information. Due to the presence of titin in both skeletal and cardiac muscle, measurement of the marker in other pathologies such as muscular dystrophies could indicate a broader use for this marker. The study also lacks immunohistochemistry and western blot data due to the lack of tissue from the enrolled patients. Despite numerous attempts to obtain 
additional western blot data using mouse tissue instead, the clone currently used could not be utilised for western blot use. Additional clones are being raised by new immunisations that could produce a clone that can be used for western blotting and immunohistochemistry. Even though the marker can be accurately measured in serum, it may be also present in other body fluids such as urine and saliva. These have not been assessed in this study due to lack of relevant material. However, when the material is made available it will be assessed accordingly. This will allow for additional assessment of the marker and the influence of renal function on its presence and levels. Measurement of the marker in a larger cohort and with additional CVD related pathologies will also allow for a better description of the marker. Measurement of additional biomarkers such as troponins and $\mathrm{CKMB}$ and direct comparisons of these with MMP-12 generated titin fragments will add to our understanding of the marker and its potential utility. Finally, even though the marker was found to be elevated in the tested clinical groups, the result represents only a "snapshot" in the complex cardiac remodeling process that occurs during pathologic events. A longitudinal study including detailed monitoring of the marker during cardiac remodeling is needed to better describe the marker, together with further assay optimisation.

\section{Conclusion}

We have developed and validated a serum-based competitive assay using a specific monoclonal antibody for the detection of the titin sequence, titin-12670 (NVTVEARLIK). To our knowledge this is the first neoepitope biomarker that specifically detects in serum, in vivo, titin remodeling activity and is related to cardiac damage in the cohorts examined in this study. The data suggest that there is a promising potential for the use of such biomarkers to assesscardiovascular related diseases in a clinical setting. Further testing in additional cohorts is required to validate our findings.

\section{Abbreviations \\ MMP: Matrix metalloproteinase; ECM: Extracellular matrix; ECMR: Extracellular matrix remodeling; LLD: Lower limit of detection; CVD: Cardiovascular disease; CABG: Coronary artery bypass graft; HCC: High coronary calcium; AMI: Acute myocardial infarction; ELISA: Enzyme linked immunosorbent assay.}

\section{Competing interests}

Efstathios Vassiliadis, Natasha Barascuk, Federica Genovese, Xiaoliang Chen, Qinlong Zheng, Diana J. Leeming and Morten A. Karsdal are full-time employees of Nordic Bioscience.

\section{Authors' contributions}

EV carried out sequence selection, ELISA development, draft manuscript, statistical analysis, tissue extractions, conceived study and study coordination. LMR provided patient samples, helped with the description of cohorts and the discussion and actively contributed to ideas for the project. IB performed the statistical analysis and added key parts of the methods, results and discussion. DVL developed the ELISA and added to the manuscript. NB carried out immunoassays, tissue extraction and drafted additions to the manuscript. RC has creatively added to the manuscript discussion and introduction. SH has collected patient samples and biochemical measurements and helped in the description of the cohorts. LS has collected patient samples and biochemical measurements and helped in the description of the cohorts. ACPD has collected patient samples and biochemical measurements and helped in the description of the cohorts. FG has performed tissue extractions, immunoassays and sections in both materials and methods and results. KLD has helped with the mass spectrometry selection process and collection of data as well as creative inputs into the manuscript. QZ has performed mice immunisations, clone selection, antibody production and early immunoassay characterisation data. XC has performed mice immunisations, clone selection, antibody production and early immunoassay characterisation data. DJL has creatively added into the manuscript building process and ELISA optimisation steps. CC has creatively added into the formation of manuscript, read and approved the final version. MK has creatively added into the ELISA optimisation steps, formation of manuscript, study coordination, read and approved the final version. All authors read and approved the final manuscript.

\section{Acknowledgement}

We acknowledge the funding from the Danish Ministry of Science, Technology and Innovation and the Den Danske Forskningsfond, Centre for Clinical and Basic Research. We would also like to thank Dr. Athanasios Didangelos from the Kings British Heart Foundation Centre, Kings College, London, UK for his insights on MMP activity in cardiac tissue.

\section{Author details}

${ }^{1}$ Nordic Bioscience A/S, Herlev Hovedgade 207, DK-2730 Herlev, Denmark. ${ }^{2}$ School of Endocrinology, University of Southern Denmark, Odense, Denmark. ${ }^{3}$ Department of Clinical Biochemistry and Pharmacology, Odense University Hospital, Odense, Denmark. ${ }^{4}$ Division of Cardiology, Hospital for Sick Children, Toronto, Canada. ${ }^{5}$ Department of Cardiology, Odense University Hospital, Odense, Denmark. ${ }^{6}$ Eli Lilly and Company, Indianapolis, IN, USA. 7 Nordic Bioscience Beijing, Beijing, China.

Received: 28 February 2012 Accepted: 15 June 2012

Published: 6 July 2012

\section{References}

1. LeWinter MM, Wu Y, Labeit S, Granzier H: Cardiac titin: structure, functions and role in disease. Clin Chim Acta 2007, 375(1-2):1-9.

2. Fukuda N, Terui T, Ishiwata S, Kurihara S: Titin-based regulations of diastolic and systolic functions of mammalian cardiac muscle. $J \mathrm{Mo} / \mathrm{Cell}$ Cardiol 2010, 48(5):876-881.

3. LeWinter MM, Granzier H: Cardiac titin: a multifunctional giant. Circulation 2010, 121(19):2137-2145.

4. Wu Y, Bell SP, Trombitas $K$, et al: Changes in titin isoform expression in pacing-induced cardiac failure give rise to increased passive muscle stiffness. Circulation 2002, 106(11):1384-1389.

5. Sutko JL, Publicover NG, Moss RL: Titin: an elastic link between length and active force production in myocardium. Circulation 2001, 104(14):1585-1587.

6. Granzier HL, Irving TC: Passive tension in cardiac muscle: contribution of collagen, titin, microtubules, and intermediate filaments. Biophys J 1995, 68(3):1027-1044.

7. Jaber WA, Maniu C, Krysiak J, et al: Titin isoforms, extracellular matrix, and global chamber remodeling in experimental dilated cardiomyopathy: functional implications and mechanistic insight. Circ Heart Fail 2008, 1(3):192-199.

8. Hein S, Gaasch WH, Schaper J: Giant molecule titin and myocardial stiffness. Circulation 2002, 106(11):1302-1304.

9. LeWinter MM: Titin isoforms in heart failure: are there benefits to supersizing? Circulation 2004, 110(2):109-111.

10. Bang ML, Centner T, Fornoff F, et al: The complete gene sequence of titin, expression of an unusual approximately $700-\mathrm{kDa}$ titin isoform, and its interaction with obscurin identify a novel Z-line to I-band linking system. Circ Res 2001, 89(11):1065-1072.

11. Freiburg A, Trombitas K, Hell W, et al: Series of exon-skipping events in the elastic spring region of titin as the structural basis for myofibrillar elastic diversity. Circ Res 2000, 86(11):1114-1121. 
12. Cazorla O, Freiburg A, Helmes M, et al: Differential expression of cardiac titin isoforms and modulation of cellular stiffness. Circ Res 2000, 86(1):59-67.

13. Linke WA: Sense and stretchability: the role of titin and titin-associated proteins in myocardial stress-sensing and mechanical dysfunction. Cardiovasc Res 2008, 77(4):637-648.

14. Chaturvedi RR, Herron T, Simmons R, et al: Passive stiffness of myocardium from congenital heart disease and implications for diastole. Circulation 2010, 121(8):979-988.

15. Thijssen $V L$, Borgers $M$, Lenders $M H$, et al: Temporal and spatial variations in structural protein expression during the progression from stunned to hibernating myocardium. Circulation 2004, 110(21):3313-3321.

16. Nagueh SF, Shah G, Wu Y, et al: Altered titin expression, myocardial stiffness, and left ventricular function in patients with dilated cardiomyopathy. Circulation 2004, 110(2):155-162.

17. Peng J, Raddatz K, Molkentin JD, et al: Cardiac hypertrophy and reduced contractility in hearts deficient in the titin kinase region. Circulation 2007, 115(6):743-751.

18. Linke WA: Titin stiffness in heart disease. Circulation 2003, 107(11):e73.

19. Neagoe C, Kulke M, del MF et al: Titin isoform switch in ischemic human heart disease. Circulation 2002, 106(11):1333-1341.

20. Makarenko I, Opitz CA, Leake MC, et al: Passive stiffness changes caused by upregulation of compliant titin isoforms in human dilated cardiomyopathy hearts. Circ Res 2004, 95(7):708-716.

21. Van HL, Borbely A, Niessen HW, et al: Myocardial structure and function differ in systolic and diastolic heart failure. Circulation 2006, 113(16):1966-1973.

22. Karsdal MA, Henriksen K, Leeming DJ, et al: Biochemical markers and the FDA Critical Path: how biomarkers may contribute to the understanding of pathophysiology and provide unique and necessary tools for drug development. Biomarkers 2009, 14(3):181-202.

23. Vassiliadis $E$, Veidal SS, Simonsen $H$, et al: Immunological detection of the type V collagen propeptide fragment, PVCP-1230, in connective tissue remodeling associated with liver fibrosis. Biomarkers 2011, 5:426-433.

24. Vassiliadis E, Larsen DV, Clausen RE, et al: Measurement of CO3-610, a Potential Liver Biomarker Derived from Matrix Metalloproteinase-9 Degradation of Collagen Type III, in a Rat Model of Reversible Carbon-Tetrachloride-Induced Fibrosis. Biomark Insights 2011, 6:49-58.

25. Vassiliadis E, Veidal SS, Barascuk N, et al: Measurement of matrix metalloproteinase 9-mediated collagen type III degradation fragment as a marker of skin fibrosis. BMC Dermatol 2011, 11:6.

26. Veidal SS, Vassiliadis E, Bay-Jensen AC, Tougas G, Vainer B, Karsdal MA: Procollagen type I N-terminal propeptide (PINP) is a marker for fibrogenesis in bile duct ligation-induced fibrosis in rats. Fibrogenesis Tissue Repair 2010, 3(1):5

27. Barascuk N, Vassiliadis E, Larsen $L$, et al: Development and validation of an enzyme-linked immunosorbent assay for the quantification of a specific MMP-9 mediated degradation fragment of type III collagen-A novel biomarker of atherosclerotic plaque remodeling. Clin Biochem 2011, 44(10-11):900-906

28. Kuznetsova T, Herbots L, Lopez B, et al: Prevalence of left ventricular diastolic dysfunction in a general population. Circ Heart Fail 2009, 2(2):105-112.

29. de Lemos JA: Biomarkers in Heart Disease. Malden, MA: Blackwell publishing and American Heart Association; 2008.

30. Jackson CE, Dalzell JR, Gardner RS: Prognostic utility of cardiac troponin in heart failure: a novel role for an established biomarker. Biomark Med 2009, 3(5):483-493.

31. Park JP, Cropek DM, Banta S: High affinity peptides for the recognition of the heart disease biomarker troponin I identified using phage display. Biotechnol Bioeng 2010, 105(4):678-686.

32. Rosalki SB, Roberts R, Katus HA, Giannitsis E, Ladenson JH, Apple FS: Cardiac biomarkers for detection of myocardial infarction: perspectives from past to present. Clin Chem 2004, 50(11):2205-2213

33. O'Brien PJ: Cardiac troponin is the most effective translational safety biomarker for myocardial injury in cardiotoxicity. Toxicology 2008, 245(3):206-218.

34. Ali MA, Cho WJ, Hudson B, Kassiri Z, Granzier H, Schulz R: Titin is a target of matrix metalloproteinase-2: implications in myocardial ischemia/ reperfusion injury. Circulation 2010, 122(20):2039-2047.
35. Linke WA: Molecular giant vulnerable to oxidative damage: titin joins the club of proteins degraded by matrix metalloproteinase- 2 .

Circulation 2010, 122(20):2002-2004.

36. Zhen EY, Brittain IJ, Laska DA, et al: Characterization of metalloprotease cleavage products of human articular cartilage. Arthritis Rheum 2008, 58(8):2420-2431.

37. Johnson JL, George SJ, Newby AC, Jackson CL: Divergent effects of matrix metalloproteinases $3,7,9$, and 12 on atherosclerotic plaque stability in mouse brachiocephalic arteries. Proc Natl Acad Sci U S A 2005, 102(43):15575-15580.

38. Combet C, Blanchet C, Geourjon C, Deleage G: NPS@: network protein sequence analysis. Trends Biochem Sci 2000, 25(3):147-150.

39. Musco G, Tziatzos C, Schuck P, Pastore A: Dissecting titin into its structural motifs: identification of an alpha helix near the $\mathrm{N}$-terminus. Biochemistry 1995, 34:553-561.

40. Gefter ML, Margulies DH, Scharff MD: A simple method for polyethylene glycol-promoted hybridization of mouse myeloma cells. Somatic Cell Genet 1977, 3(2):231-236.

41. Diederichsen AC, Sand NP, Norgaard B, et al: Discrepancy between coronary artery calcium score and HeartScore in middle-aged Danes: the DanRisk study. Eur J Cardiovasc Prev Rehabil 2011.

42. Lambert JM, Lopez EF, Lindsey ML: Macrophage roles following myocardial infarction. Int J Cardiol 2008, 130(2):147-158.

43. Manolio T: Novel risk markers and clinical practice. N Engl J Med 2003, 349(17):1587-1589.

44. Stampfer MJ, Ridker PM, Dzau VJ: Risk factor criteria. Circulation 2004 109(25 Suppl 1):IV3-IV5.

45. Orn S, Manhenke C, Squire IB, Ng L, Anand I, Dickstein K: Plasma MMP-2, MMP-9 and N-BNP in Long-Term Survivors Following Complicated Myocardial Infarction: Relation to Cardiac Magnetic Resonance Imaging Measures of Left Ventricular Structure and Function. Journal of Cardiac Failure 2007, 13(10):843-849.

46. Riches K, Morley ME, Peers C, Porter KE: Hypoxic inhibition of MMP-2 activation and invasion in human cardiac myofibroblasts. $\mathrm{J} \mathrm{Mol} \mathrm{Cell}$ Cardiol 2008, 44(4):737-738

47. Bellosta S, Gomaraschi M, Canavesi M, et al: Inhibition of MMP-2 activation and release as a novel mechanism for HDL-induced cardioprotection. FEBS Letters 2006, 580(25):5974-5978.

48. Turner NA, Aley PK, Hall KT, et al: Simvastatin inhibits TNF[alpha]-induced invasion of human cardiac myofibroblasts via both MMP-9-dependent and -independent mechanisms. J Mol Cell Cardiol 2007, 43(2):168-176.

49. Zhou HZ, Ma X, Gray MO, et al: Transgenic MMP-2 expression induces latent cardiac mitochondrial dysfunction. Biochem Biophysical Res Comm 2007, 358(1):189-195.

50. Wohlschlaeger J, Stubbe HD, Schmitz KJ, et al: Roles of MMP-2/-9 in cardiac dysfunction during early multiple organ failure in an ovine animal model. Pathology Res Prac 2005, 201(12):809-817.

51. Nichol JW, Engelmayr J, Cheng M, Freed LE: Co-culture induces alignment in engineered cardiac constructs via MMP-2 expression. Biochem Biophysical Res Comm 2008, 373(3):360-365.

52. Luttun A, Lutgens $E$, Manderveld A, et al: Loss of matrix metalloproteinase-9 or matrix metalloproteinase-12 protects apolipoprotein E-deficient mice against atherosclerotic media destruction but differentially affects plaque growth. Circulation 2004, 109(11):1408-1414.

53. Vanhoutte D, Heymans S: TIMPs and cardiac remodeling: [']Embracing the MMP-independent-side of the family'. J Mol Cell Cardiol 2010, 48(3):445-453.

54. Curci JA, Liao S, Huffman MD, Shapiro SD, Thompson RW: Expression and localization of macrophage elastase (matrix metalloproteinase-12) in abdominal aortic aneurysms. J Clin Invest 1998, 102(11):1900-1910.

55. Johnson JL, Devel L, Czarny B, et al: A selective matrix metalloproteinase-12 inhibitor retards atherosclerotic plaque development in apolipoprotein E-knockout mice. Arterioscler Thromb Vasc Biol 2011, 31(3):528-535.

56. Didangelos A, Yin X, Mandal $K$, et al: Extracellular matrix composition and remodeling in human abdominal aortic aneurysms: a proteomics approach. Mol Cell Proteomics 2011, 10(8):M111.

57. Liang J, Liu E, Yu Y, et al: Macrophage metalloelastase accelerates the progression of atherosclerosis in transgenic rabbits. Circulation 2006, 113(16):1993-2001.

58. Ikonomidis JS, Jones JA, Barbour JR, et al: Expression of matrix metalloproteinases and endogenous inhibitors within ascending aortic 
aneurysms of patients with Marfan syndrome. Circulation 2006, 114(1 Suppl):1365-1370.

59. Nar H, Werle K, Bauer MMT, Dollinger H, Jung B: Crystal structure of human macrophage elastase (MMP-12) in complex with a hydroxamic acid inhibitor. J Mol Biol 2001, 312(4):743-751.

60. Benoit M, Thuny F, Le PY, et al: The transcriptional programme of human heart valves reveals the natural history of infective endocarditis. PloS One 2010, 5(1):e8939.

61. Munoz-Luque J, Ros J, Fernandez-Varo G, et al: Regression of fibrosis after chronic stimulation of cannabinoid $\mathrm{CB} 2$ receptor in cirrhotic rats. J Pharmacol Exp Ther 2008, 324(2):475-483.

doi:10.1186/1479-5876-10-140

Cite this article as: Vassiliadis et al:: Clinical evaluation of a matrix metalloproteinase-12 cleaved fragment of titin as a cardiovascular serological biomarker. Journal of Translational Medicine 2012 10:140.

\section{Submit your next manuscript to BioMed Central and take full advantage of:}

- Convenient online submission

- Thorough peer review

- No space constraints or color figure charges

- Immediate publication on acceptance

- Inclusion in PubMed, CAS, Scopus and Google Scholar

- Research which is freely available for redistribution 